\title{
Testing approximate predictions of displacements of cosmological dark matter halos
}

\section{Emiliano Munari, ${ }^{a, b}$ Pierluigi Monaco, ${ }^{a, b, c}$ Jun Koda, ${ }^{d}$ Francisco-Shu Kitaura, ${ }^{e}$ Emiliano Sefusatti, ${ }^{b}$ Stefano Borgani ${ }^{a, b, c}$}

${ }^{a}$ Department of Physics, Astronomy Unit, University of Trieste, via Tiepolo 11, I-34143 Trieste, Italy

${ }^{b}$ INAF-Osservatorio Astronomico di Trieste, via Tiepolo 11, I-34143 Trieste, Italy

${ }^{c}$ INFN, Sezione di Trieste

${ }^{d}$ Dipartimento di Matematica e Fisica, Universitá degli Studi Roma Tre, via della Vasca Navale 84, I-00146 Roma, Italy

${ }^{e}$ Leibniz-Institut fur Astrophysik Potsdam (AIP), An der Sternwarte 16, D-14482 Potsdam, Germany

E-mail: munari@oats.inaf.it, monaco@oats.inaf.it

\begin{abstract}
We present a test to quantify how well some approximate methods, designed to reproduce the mildly non-linear evolution of perturbations, are able to reproduce the clustering of DM halos once the grouping of particles into halos is defined and kept fixed. The following methods have been considered: Lagrangian Perturbation Theory (LPT) up to third order, Truncated LPT, Augmented LPT, MUSCLE and COLA. The test runs as follows: halos are defined by applying a friends-of-friends (FoF) halo finder to the output of an Nbody simulation. The approximate methods are then applied to the same initial conditions of the simulation, producing for all particles displacements from their starting position and velocities. The position and velocity of each halo are computed by averaging over the particles that belong to that halo, according to the FoF halo finder. This procedure allows us to perform a well-posed test of how clustering of the matter density and halo density fields are recovered, without asking to the approximate method an accurate reconstruction of halos. We have considered the results at $z=0,0.5,1$, and we have analysed power spectrum in real and redshift space, object-by-object difference in position and velocity, density Probability Distribution Function (PDF) and its moments, phase difference of Fourier modes.

We find that higher LPT orders are generally able to better reproduce the clustering of halos, while little or no improvement is found for the matter density field when going to 2LPT and 3LPT. Augmentation provides some improvement when coupled with 2LPT, while its effect is limited when coupled with 3LPT. Little improvement is brought by MUSCLE with respect to Augmentation. The more expensive particle-mesh code COLA outperforms all LPT methods, and this is true even for mesh sizes as large as the inter-particle distance. This test sets an upper limit on the ability of these methods to reproduce the clustering of halos, for the cases when these objects are reconstructed at the object-by-object level.
\end{abstract}




\section{Contents}

1 Introduction 1

2 Approximate methods $\quad 3$

3 Matter and halo catalogues $\quad 5$

4 Comparison of methods $\quad 5$

4.1 Computational resources $\quad 7$

$\begin{array}{lll}4.2 & \text { Halo positions and velocities at the object-by-object level } & 7\end{array}$

4.3 Power spectrum in real and redshift space 8

4.4 Further probes 9

5 Conclusions

\section{Introduction}

The very precise measurement of temperature fluctuations of the Cosmic Microwave Background has yielded tight constraints on the cosmological parameters [1], but this accuracy has provided no further clues on the nature of the postulated Dark Matter (DM) and dark energy components. Further tests, able to shed light on these components and especially to constrain the equation of state of dark energy, require accurate measurements of fluctuations at lower redshift, where galaxies are the main tracers of the density field, both through their clustering and the deformation of their images subject to gravitational lensing. However, these measurements are much more difficult to interpret, due to the highly non-linear character of the density peaks that host galaxies, usually referred to as DM halos. As statistical errors will be beaten down by the large number of objects of future surveys, like e.g. eBOSS ${ }^{1}$ [Extended Baryon Oscillation Spectroscopic Survey 2], DESI ${ }^{2}$ [Dark Energy Spectroscopic Instrument 3], $\mathrm{LSST}^{3}$ [Large Synoptic Survey Telescope 4], or ESA's Euclid ${ }^{4}[5]$, the control of systematics will become of huge importance. This, together with the determination of accurate covariance matrices of observables, requires usage of a large number of mock galaxy catalogs. The high level of accuracy needed to match these observations can be reached only by N-body simulations [6-8]. However, simulating a very large volume, with enough resolution to sample the DM halos that host the faintest and most numerous galaxies observed in a typical survey, is a challenge for N-body codes [e.g. 9]. Running thousands of such simulations has a prohibitive cost in terms of computing time.

A possible alternative to greatly reduce the cost of these simulations, recently reviewed in [10], is to resort to approximate methods that are able to provide catalogs of DM halos with clustering properties that are accurate down to a scale of order of a few Mpc. This is the scale at which clustering starts to be dominated by the 1-halo term of galaxies that live in the same DM halo, and at this scale the complicated and poorly known physics of

\footnotetext{
${ }^{1}$ http://www.sdss.org/surveys/eboss/

${ }^{2}$ http://desi.lbl.gov/

${ }^{3}$ http: //www. lsst.org/

${ }^{4}$ http://sci.esa.int/euclid/
} 
baryons makes predictions much more uncertain. Usage of approximate methods amounts to compromising accuracy in favour of speed, and the gain in computing time can easily be of a factor of $\sim 1000$. Then, the most promising strategy to face the problem of massive production of mock catalogs is a mixed one, with few very accurate mocks from N-body simulations and thousands based on approximate methods. As a consequence, and somewhat counter-intuitively, high-precision cosmology has lead to renewed attention on approximate methods.

Focusing on the production of catalogs of DM halos to predict galaxy clustering, approximate methods are required to solve two problems. (1) They have to produce a good approximation of the large-scale displacement field of matter from its initial position to the position at the observation redshift. This yields predictions of the matter density and the peculiar velocity fields as well. (2) They have to specify how to populate the large-scale density field with a distribution of biased DM halos, compatible with the result of an N-body simulation. Problem (2) is faced with two main strategies, outlined in [10]. The first strategy consists in sampling the density field on a Lagrangian grid, and group grid points, alias particles, into DM halos so as to achieve a good object-by-object agreement with simulations. The methods that follow this strategy are called Lagrangian methods. The second strategy, pursued by the bias-based methods, consists in starting from the mildly non-linear density field sampled on a $\sim \mathrm{Mpc}$ grid, then Monte-Carlo generate a distribution of halos by implementing a sophisticated bias scheme, that will be calibrated on a large N-body simulation.

Problem (2), populating a large-scale density field with DM halos, is much more demanding to solve than problem (1), generating the large-scale density and velocity fields, because it requires to have some control of the highly non-linear regime of DM halos. Yet, the two problems have different impacts on the reproduction of halo clustering: the large-scale displacements determine the smallest scales at which halo clustering is correctly recovered, while halo reconstruction determines the mass function of DM halos and their bias, or in other terms the clustering amplitude.

A number of different approximations, that will be mentioned in the following, have been put forward to predict the large-scale density field that are valid in the mildly nonlinear regime, and their ability to predict the matter density field has been throughly tested and quantified by each proponent. A comprehensive comparison of how methods based on these approximations are able to reproduce the clustering of halos, in real and redshift space, has been presented by [11]. However, a straight comparison of methods that solve both the two problems mentioned above leads to results that are difficult to interpret. As an example, the bias-based EZmocks code [12] uses ZA to produce the matter density field, and ZA is known to poorly reproduce power at $k \gtrsim 0.1 \mathrm{~h} / \mathrm{Mpc}$; however, that method recovers much of that power by suitably calibrating its bias scheme. Conversely, a comprehensive comparison of the ability of methods that solve the first problem, predict the displacement field, to predict the clustering of DM halos has not been presented so far.

In this paper we perform a test on the accuracy of approximate methods, designed to predict the displacement field of particles, in reproducing halo clustering once the grouping of particles into halos is defined and kept fixed for all methods. We have taken an N-body simulation, run a standard friends-of-friends halo finder to define halos, then have used a number of methods to create the displacement field, given the same initial conditions as the simulation. Halo positions and velocities have been computed as the center of mass, and average velocity, of all particles that are known to belong to that halo. This allows us to perform a well-posed test of how clustering of the matter density and halo density fields are 
recovered. The value of this test is to provide an upper limit to the ability of an approximated method of the Lagrangian class defined above to reproduce halo clustering.

The paper is organized as follows. Section 2 gives a list and a quick description of the methods used in this paper. In particular, we will test LPT up to third order [13-15], Truncated LPT [16, 17], Augmented LPT [18], MUSCLE [19] and COLA [20, 21]. Section 3 describes the simulation set-up. Positions and velocities of DM halos are tested at the objectby-object level in Section 4.2. In Section 4.3 the clustering is investigated, while in Section 4.4 other probes of the halo distributions are carried out. The computational resources needed by the different methods are compared in Section 4.1. Results are summarized and conclusions are drawn in Section 5.

\section{Approximate methods}

In this paper we test implementations of the following approximate methods. A more comprehensive description is given in [10].

(i) LPT is based on the Lagrangian description of fluid dynamics, where the evolution of the cosmic fluid is recasts into a map from the initial position $\vec{q}$ of the fluid element to the final position $\vec{x}(\vec{q})$ as:

$$
\vec{x}(\vec{q}, t)=\vec{q}+\vec{S}(\vec{q}, t)
$$

To first order, the growing mode of the displacement field $\vec{S}$ is equal to the ZA:

$$
\vec{S}(\vec{q}, t)=-D(t) \nabla_{\vec{q}} \phi(\vec{q})
$$

where $D(t)$ is the linear growing mode and $\phi$ is the (suitably rescaled) peculiar gravitational potential. Second-order LPT (hereafter 2LPT) can be written as the sum of ZA and a secondorder term; this can be factorized into a time function $D^{(2)}(t)$, of the order of $D^{2}$, and the gradient of a second-order potential $\phi^{(2)}(\vec{q})$, that can be found by solving a Poisson equation. Third-order LPT (hereafter 3LPT) adds one more contribution that can be written as the sum of three terms, each of them factorisable as the product of a time-dependent and a space-dependent function. The time-dependent functions are of order $D^{3}$. Two of the three space-dependent functions can be obtained as the gradient of a potential, that obeys a Poisson equation, and are thus irrotational as the previous terms, while the third can be obtained as the curl of a vector potential, and is purely rotational. This third term is small and is commonly neglected; it will be neglected in the following.

(ii) The Truncated Zeldovich Approximation (hereafter TZA) was proposed by [16] in order to limit the effect of orbit-crossing taking place at small scales. It consists in applying ZA to a filtered version of the linear density field; a Gaussian shape is typically assumed for the filter and the radius is computed as that for which the standard deviation of the smoothed density is equal to unity. The extension to T2LPT and T3LPT (with obvious meaning of the acronyms) has been presented by [17]; a review of the various truncation schemes is given by [22].

(iii) Augmented LPT (hereafter ALPT) was proposed by [18] as a way to limit orbit crossing at small scales, while preserving small-scale power. The starting point [23] is that the divergence of the displacement field $\nabla_{\vec{q}} \cdot \vec{S}(\vec{q})$, called stretching parameter, levels to -3 inside bound structures. This stretching parameter can be approximated by the following formula based on spherical collapse, that is found to be a good fit to simulations: 


$$
\nabla_{\vec{q}} \cdot \vec{S}(\vec{q})= \begin{cases}3\left[\left(1-\frac{2}{3} D(t) \delta(\vec{q})\right)^{1 / 2}-1\right] & \text { if } \delta<\frac{3}{2 D} \\ -3 & \text { if } \delta \geq \frac{3}{2 D}\end{cases}
$$

ALPT is based on separating large-scale and small-scale modes, modeling the formers with LPT and the latters with the solution of equation 2.3 , that we will call $\vec{S}_{\mathrm{SC}}$. Using a Gaussian kernel $\kappa(\vec{q} ; R)$ to perform this separation:

$$
\vec{S}_{\mathrm{ALPT}}(\vec{q})=\kappa(\vec{q} ; R) * \vec{S}_{\mathrm{LPT}}+[1-\kappa(\vec{q}, R)] * \vec{S}_{\mathrm{SC}}
$$

We will apply augmentation to 2LPT (A2LPT) and 3LPT (A3LPT). The radius $R$ for the smoothing is set to $4 \mathrm{Mpc} / \mathrm{h}$ for A2LPT and $1.25 \mathrm{Mpc} / \mathrm{h}$ for A3LPT at $z=0$. These values have been chosen to maximize the performance in the halo catalog power spectrum in real space at $z=0$. The values of the smoothing radius are changed with redshift by solving the following equation:

$$
\sigma\left(z ; R_{z}\right)=\sigma\left(z=0 ; R_{z=0}\right)
$$

where $\sigma(z ; R)$ is the standard deviation of the density field at redshift $z$ when smoothing the field with a Gaussian filter on a scale $R$.

(iv) MUSCLE [19] implements a refined prescription for the spherical collapse. We apply this method for modeling the small-scale modes, using 2LPT for the large-scale ones, in the same way done for ALPT. Similarly to the augmented LPT, the stretching parameter is computed as in eq. 2.3, with the further requirement that $G_{R}(\delta(\vec{q}))<3 / 2 D$ for each $R \geq R_{i p}$, where $G_{R}$ indicates a Gaussian smoothing of the density field with scale radius $\mathrm{R}$, and $R_{i p}$ is the inter-particle distance which, in our configuration, is equal to $1 \mathrm{Mpc} / \mathrm{h}$. We have adopted 8 linearly spaced smoothing radii, from $1 \mathrm{Mpc} / \mathrm{h}$ to $57 \mathrm{Mpc} / \mathrm{h}$. The ALPT radius for connecting the small and large scales is set to $4 \mathrm{Mpc} / \mathrm{h}$ at $z=0$, and scaled with redshift according to eq. 2.5.

(v) Particle-mesh (PM) codes have recently become very popular as a tool to produce quick simulations. PM [24] consists in solving the Poisson equation on a mesh, using Fast Fourier Transforms (FFTs) to speed up the computation. This is a standard N-body technique, often used in conjunction with the tree algorithm [25] to better integrate the large-scale modes and speed-up the evolution from the initial conditions. Strictly speaking, PM codes are N-body codes, but their accuracy is severely limited by the size of the non-adaptive mesh, so we place them in the class of approximate methods. But a PM code is in principle able to accurately recover clustering down to the mesh scale if a sufficient number of time-steps is adopted, so it is obvious that it will outperform all LPT-based methods. This comes at a cost: the performance of a PM code will depend on the number of time-steps and on the mesh size.

When few time-steps are used, the standard PM code does not correctly reproduce the growing mode. Two methods have been proposed to enhance the quick convergence of these methods with the number of time-steps. In COmoving Lagrangian Acceleration [COLA, 20] the gravitational force is computed in a gauge that is comoving with the $2 \mathrm{LPT}$ solution, thus guaranteeing that the growing mode is correctly reproduced (at the second order in this case). In FastPM [26] the kick and drift operators of PM are redefined, so that the velocity is not assumed to be constant within a time-step but to evolve as predicted by ZA. In this paper we will test only the parallel implementation of COLA developed by [21] (other parallel versions are due to $[27,28])$. Indeed, the aim of the paper is not to test the performance of different PM implementations, something that has already been done in the papers cited above, but to 
compare the results of LPT-based methods with at least one state-of-the-art PM code. For this paper, COLA has been run with 10 time-steps with $\Delta a=0.1, a$ being the scale factor. We will test here in particular the performance of COLA when changing mesh size: this is the main parameter that makes PM codes slower and more memory consuming than LPT-based ones, but a fine mesh is only required to correctly identify DM halos, something that is not needed in our context.

In Fig. 1 we show density maps obtained for the different methods.

\section{Matter and halo catalogues}

Throughout this paper we use the following setup: $1024^{3}$ particles in a $1024 \mathrm{Mpc} / \mathrm{h}$ side box with $\Omega_{0}=0.25, \Omega_{\Lambda}=0.75, \Omega_{b}=0.044, h=0.7$, and $\sigma_{8}=0.8$. With this configuration, the particle mass is $6.9 \times 10^{10} \mathrm{M}_{\odot} / h$.

To compute the matter and halo distributions, we proceed as follows:

1. We set up the initial conditions (ICs) as a set of particles distributed on a regular grid, labeling them with an ID that is relative to the position in the grid. This is the same set up used in the N-body simulations before the small perturbations are generated.

2. Starting from these ICs, we run an N-body simulation with the GADGET 3 [29] code (the same used in [30]). 1/50 of the mean inter-particle distance is adopted for the Plummer-equivalent gravitational softening. In this way, we obtain the distribution of particles at $\mathrm{z}=0,0.5$ and 1 . We create halo catalogues at these redshifts, and the lists of particles belonging to each halo, by running a standard friends-of-friends (FOF) algorithm, adopting a constant linking length equal to 0.2 times the inter-particle distance. All haloes with more than 32 particles are considered as faithfully reconstructed.

3. We consider the ICs described at point 1 and generate a perturbation field in the same way as is done for the N-body, and displace the particles to $\mathrm{z}=0,0.5$ and 1 by means of an approximate method. The following methods are used: Zel'dovich approximation (ZA), 2LPT, 3LPT, Truncated Zel'dovich (TZA), Truncated 2LPT (T2LPT), Augmented 2LPT (A2LPT), Augmented 3LPT (A3LPT), MUSCLE with 2LPT, and COLA with 4 different mesh sizes.

4. Since we already know to which halo each particle belongs to, as explained in point 2 , we compute the center of mass's position and velocity of each halo by averaging over its displaced particles.

The final result is the matter (particles) distribution and the halo catalogue for each realization. We can therefore compare these distributions to that of the N-body simulation.

In the following, we will denote as $N$-body halos those obtained running the FoF algorithm on the N-body simulation, while we will denote as method halos those obtained from the same particles, displaced according to the different methods. For COLA, halo clustering has been found to be insensitive to the mesh size, so we will mostly show the 1024 mesh case, with the exception of the matter power spectra were we give results for all the 4 mesh sizes.

\section{Comparison of methods}

After having assessed the cost of the various methods in terms of computing time and memory, we will first consider how halo positions and velocities are recovered at an object-by-object 


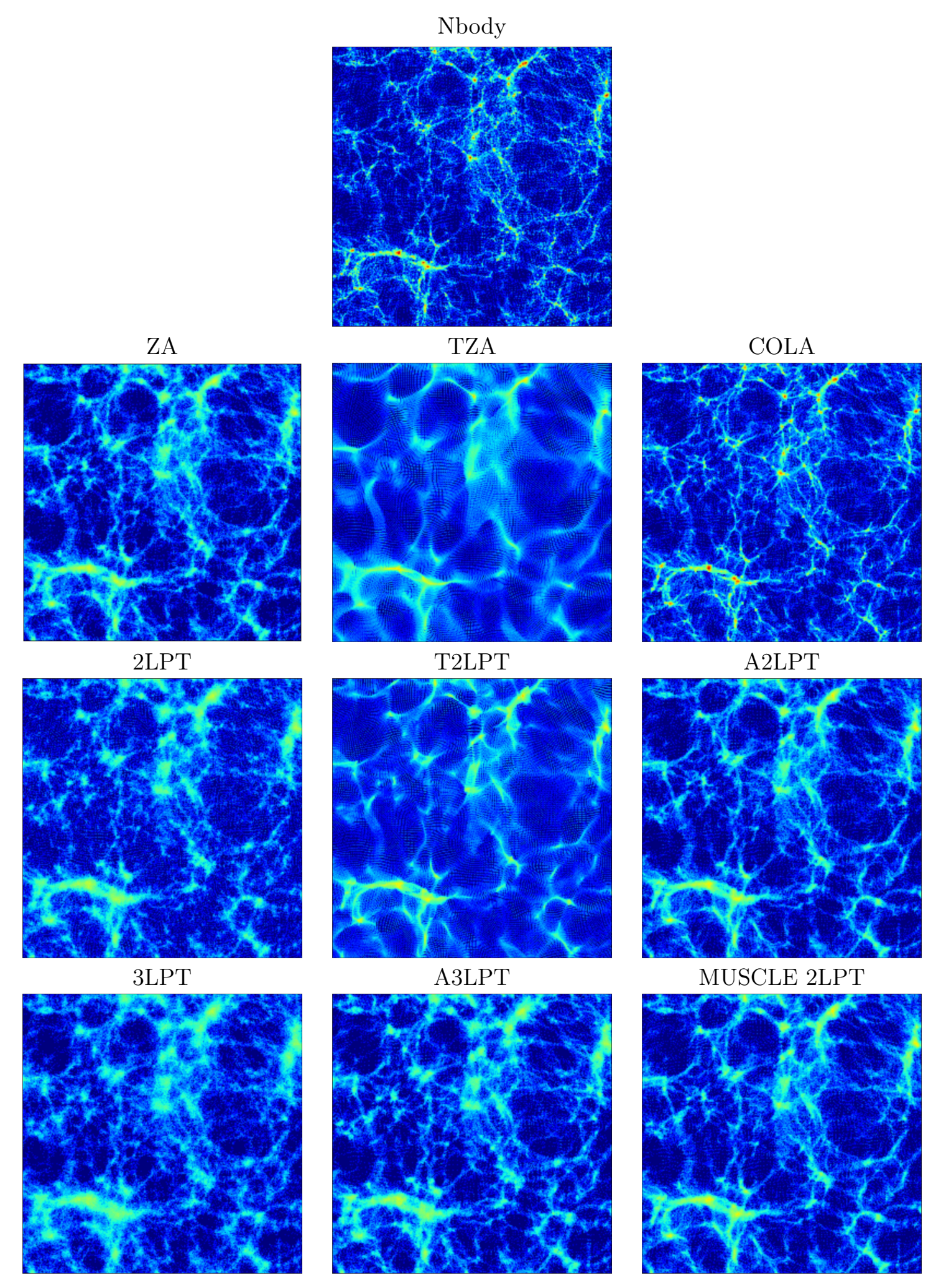

Figure 1. Density maps (logarithmic color scale) of $10 \mathrm{Mpc} / \mathrm{h}$ depth and $200 \mathrm{Mpc} / \mathrm{h}$ side slices of the different realizations at $z=0$. 


\begin{tabular}{r|rc} 
& CPU Time (s) & Memory (byte/part) \\
\hline ZA & 655 & 48 \\
TZA & 805 & 48 \\
2LPT & 1918 & 96 \\
T2LPT & 2116 & 96 \\
A2LPT & 5103 & 104 \\
3LPT & 3803 & 144 \\
A3LPT & 7099 & 152 \\
MUSCLE 2LPT & 11707 & 104 \\
\hline COLA (nc3072) & 47674 & 257 (single precision) \\
COLA (nc2048) & 15888 & 151 (single precision) \\
COLA (nc1024) & 5171 & 113 (single precision) \\
COLA (nc512) & 3636 & 106 (single precision)
\end{tabular}

Table 1. CPU time (wall-clock time $\times$ number of cores) and memory needed by the different runs. All the runs except COLA are in double precision.

level. We will then address the matter and halo power spectra in real space, and the first two moments, monopole and quadrupole, of the halo power spectra in redshift space. We will finally address other probes that are sensitive to higher moments, by computing the halo number density on a grid and quantifying the difference of phases of the density Fourier transforms and the density PDFs. We will finally quantify the moments of the density PDFs as a function of the grid size used to compute the density.

\subsection{Computational resources}

We provide estimates of the computational resources needed by each method. For this, we consider the part of the code where the displacements actually take place, therefore without accounting for the time and memory needed for initialization, for writing the results, or for any other post-processing analysis. All the LPT-based methods have been run on the Galileo machine at CINECA $^{5}$ on 64 cores, while COLA runs have been carried out at the Green II super computer at Swinburne University of Technology ${ }^{6}$ using 64 cores (128 for nc3072). COLA is run in single-precision, while for all the other methods the double-precision is adopted. Clearly, a consistent comparison can be done among LPT-based methods and among COLA runs, but the comparison of the two sets, run on very different machines, must be taken as indicative.

In Tab. 1 we report the time (wall-clock $\times$ number of cores) and the memory needed by the different runs.

\subsection{Halo positions and velocities at the object-by-object level}

Fig. 2 shows, for the three redshifts, the goodness of the prediction of position and velocity of method halos, at the object-by-object level. Calling $\Delta X, \Delta Y$, and $\Delta Z$ the distances, along the three axes, of method halos from the corresponding $N$-body halos, in the top row of the figure we show the quantity $\sqrt{\left\langle(\Delta X)^{2}+(\Delta Y)^{2}+(\Delta Z)^{2}\right\rangle}$, in units of the interparticle distance (that is $1 h^{-1} \mathrm{Mpc}$ ). Calling $|V|$ the magnitude of the velocity of method halos, and $\left|V_{\text {sim }}\right|$ that of the corresponding $N$-body halos, in the second and third row of the figure

\footnotetext{
${ }^{5}$ http://www.cineca.it/en/content/galileo

${ }^{6}$ http://astronomy.swin.edu.au/supercomputing/green2/
} 
we show the median values and the dispersion of $|V| /\left|V_{\text {sim }}\right|$. In the last row we show the median value of the angle between the velocity vector of method halos and $N$-body halos, $\vec{V} \cdot \overrightarrow{V_{\text {sim }}} /|V|\left|V_{\text {sim }}\right|$.

From this figure it is clear that, independently of the redshift, higher LPT orders better reproduce all the statistics shown, with a gradual increase of accuracy at higher redshift. Both truncation and, to a lesser extent, augmentation appear to worsen the performance of LPT, especially for low mass halos; however, the augmented version of 3LPT, A3LPT, shows only little difference from the pure 3LPT. MUSCLE appears to perform as good as A2LPT. Overall, COLA provides the most accurate results, with average differences in position amounting to less than $10 \%$ of the inter-particle distance, and velocities accurate to within a few per cent (and aligned to within 1-2 degrees).

\subsection{Power spectrum in real and redshift space}

In Fig. 3 we show the power spectrum (computed including aliasing reduction, as described in [31]) in real space for both the matter field (left panels) and the halo catalogues (right panels) generated with the different methods, at $\mathrm{z}=0,0.5$, and 1 (top, middle and bottom panels, respectively). In each panel, we show the power spectrum and the ratio with that on the N-body. As for the matter field, higher orders of LPT (2LPT and 3LPT) give very little improvement with respect to ZA, and the truncation does not bear any significant improvement (T2LPT) or even worsens the performance (TZA) with respect to the standard LPT versions. Augmentation provides some noticeable improvements in the case of 2LPT. Interestingly, A2LPT drops below the 10\% accuracy at higher wavelengths than A3LPT. MUSCLE provides minor improvements with respect to A2LPT at $z=0$, becoming indistinguishable from A2LPT at higher redshifts. As expected, COLA outperforms the LPT methods in the matter power spectrum, but its accuracy drops below $10 \%$ well before $k=1 \mathrm{~h} / \mathrm{Mpc}$. There is no significant difference among the COLA runs with different mesh size, except for the coarsest one, that loses power at smaller $\mathrm{k}$ with respect to the finer meshes.

When considering the halo power spectrum, most methods show a significantly better level of agreement with the simulation. The gain in going to higher LPT orders becomes very evident in this case, with 3LPT being better than $10 \%$ accurate for $k<0.5-0.7 h / \mathrm{Mpc}$, higher values referring to higher redshift. The truncated versions always give worse results than the corresponding straight LPT order. Similarly to what happens in the matter density power spectra, the augmentation brings noticeable improvements only when coupled with 2LPT; MUSCLE and A2LPT show similar performance, while 3LPT and A3LPT are very similar. The accuracy of COLA improves as well, with most mesh sizes (but the coarsest one) being better than $10 \%$ accurate almost down to $k=1 \mathrm{~h} / \mathrm{Mpc}$, outperforming again LPT-based methods. This time, COLA runs with varying meshes show some difference in the halo power spectrum, and the 1024 mesh performs as good as, or even better than, the finer meshes, that sometimes give spurious power at high wavenumbers.

In Fig. 4 we show the monopole (left panels) and quadrupole (right panels) of the power spectrum in redshift space, at the three reference redshifts; we limit this analysis to the halo catalogues. The monopole $P_{0}(k)$ gives the same qualitative results of $P(k)$, so that all conclusions drawn above hold here as well. Noticeably, the agreement of methods and simulations improves at high redshift, so that 3LPT and A3LPT result $1 \%$ accurate up to $k=0.5-0.6 \mathrm{~h} / \mathrm{Mpc}$ at $z=1$; this improvement is lost at $z=0$, where LPT-based methods lose more power than in the real space. The same is true for COLA, where improvement with respect to real space is seen also at $z=0$. 
The quadrupole has a different behaviour. At $z=0.5$ and $z=1$ most methods tend to overestimate the quadrupole of the N-body halos at high wavenumbers. LPT-based methods (and some COLA configurations at $z=1$ ) show a minimum at some $k$, followed by a rise, so the $10 \%$ accuracy level is set by the overestimation trend. Overall, the accuracy with which the quadrupole is recovered is definitely worse than for the monopole, although the sequence of underestimation and overestimation mitigate this difference, giving a relatively high wavenumber below which $10 \%$ accuracy is reached. At $z=0$ the underestimation dominates, and even the best LPT-methods are $10 \%$ accurate only at $k<0.2 h / \mathrm{Mpc}$. COLA shows a relatively more stable behaviour, with the 1024 mesh configuration giving again the most stable results.

\subsection{Further probes}

We now concentrate on other quantifications of the density field that are sensitive to higher order statistics; these are affected by shot noise for the relatively low statistics that we are considering here. For each halo catalogue we have computed the density field of halos $\rho_{h}$ by adopting a count in cell (CIC) algorithm on a $150^{3}$ cell grid. As a first probe, we Fouriertransform the density field and compute, for each Fourier mode, the difference between the phases in the simulation and in each displacement method. For all the runs, the median phase difference is compatible with 0 , as expected, so we give a quantification of the variance between phases in bins of $k$. Fig. 5 shows the standard deviation of the phase difference between the N-body and the various method catalogues. Phase correlations are always significant, the standard deviation being much smaller than $\pi$. At higher redshift the variance gets always lower, as the Universe is less non-linear and the approximate displacements are therefore more accurate. Higher LPT orders present smaller variance at all wavelengths. Truncation, augmentation and MUSCLE worsen again the performance with respect to the standard counterparts. COLA gives a better agreement with simulations, but not by a large factor; at $z=1$ is comparable with $3 \mathrm{LPT}$.

We then extend the computation of the density field to a varying number of grid cells, from $50^{3}$ to $150^{3}$, corresponding to cell sizes of $\sim 30 \mathrm{Mpc}$ down to $\sim 10 \mathrm{Mpc}$. For each grid size, we compute the density contrast as $\delta_{h}=\left(\rho_{h}-\left\langle\rho_{h}\right\rangle\right) /\left\langle\rho_{h}\right\rangle$, where $\left\langle\rho_{h}\right\rangle$ is the average density, computed as the total number of halos divided by the volume of the whole box. Fig. 6 shows the PDFs of this density contrast, for the $100^{3}$ cell grid at $z=0$. The lower panel gives the ratio of method PDFs with respect to the N-body one. As expected, the different methods are efficient at recovering the PDF for low and intermediate density regions, while they lose power at high density. Higher orders of LPT again provide a more accurate recovery of the PDF. This time, A2LPT and MUSCLE provide a better recovery, with respect to the pure 2LPT, of the high-density tail of the PDF, while augmentation brings no apparent advantage with respect to the standard 3LPT. COLA is the only method able to recover the high-density $\mathrm{PDF}$, though with significant noise.

To better compare the differences of the halo distributions, we compute their moments (variance, skewness and kurtosis). These are shown in Fig. 7 for the three redshifts and for the different grids. Again, higher LPT orders recover more accurately the moments of the PDF. 3LPT provides an excellent recovery of the variance, with differences of order $\sim 1 \%$ or less, with its augmented version not yielding appreciable improvements. Augmentation brings instead noticeable improvements to the 2LPT performance, with differences of order $\sim 2 \%$ and a weak dependence on the grid size. MUSCLE brings no improvements at high redshift, and $\mathrm{a} \sim 1 \%$ at low redshift with respect to A2LPT, with a weak dependence on the grid size. 
3LPT and its augmented version underestimate the skewness by $\sim 5 \%$ at $z=0$, improving to $\sim 1-3 \%$ at higher redshift. $2 \mathrm{LPT}$ is $\sim 5 \%$ lower than $3 \mathrm{LPT}$, but its augmented version, as well as MUSCLE, perform considerably better. 3LPT and A3LPT are below $10 \%$ difference in the recovery of kurtosis only at high redshift, reaching differences of up to $\sim 20 \%$ at $z=0$. For 2LPT, the augmentation and MUSCLE bring improvements only at high redshift, while it is even worse than the standard 2LPT at $z=0$, even though this depends on the grid size.

The improved performance of higher order LPT is not appreciable from Fig. 1, where structures get fuzzier as the LPT order increases. To better illustrate this improvement, in Fig. 8 we show a $100 \times 100 \times 10 \mathrm{Mpch}^{-1}$ slice of the density field of the ZA, 3LPT, COLA, and A3LPT realizations. Superimposed are the density levels of the N-body realization corresponding to a density threshold equal to 1.5 times the mean density, smoothed with a Gaussian kernel with $\sigma=333 \mathrm{kpch}^{-1}$. From this figure it is possible to appreciate the effect of increasing the LPT order. 3LPT is able to reproduce the large-scale structure more accurately than ZA: this is particularly evident in the filaments, that appear offset in the ZA field, and better located in the 3LPT and A3LPT ones.

\section{Conclusions}

We have considered the outputs at $z=0,0.5,1$ of an N-body simulation, and run a FOF halo finder to define halos. Using the same initial conditions, we have generated displacement fields at the three reference redshifts using the following approximate methods: Lagrangian Perturbation Theory (LPT) methods up to third order, Truncated LPT, Augmented LPT, MUSCLE and COLA. Using the membership given by the FOF run on the simulation to associate particles to halos, we have obtained halo positions and velocities in the different realizations of the approximate methods. This has allowed us to test how clustering of the matter density and halo density fields are recovered, without relying on the approximate method themselves to reconstruct the halos. We have analysed power spectrum in real and redshift space, object-by-object difference in position and velocity, density Probability Distribution Function and its moments, and phase difference of Fourier modes.

The main results are the following:

- The higher the LPT order, the more similar the results are to the N-body. In the power spectrum the improvement is marginal when considering the matter density field, while it is clear for the halos, with 3LPT being better than $10 \%$ accurate for $k<0.5-0.7$ $\mathrm{h} / \mathrm{Mpc}$ in real space, and $1 \%$ accurate up to $k=0.5-0.6 \mathrm{~h} / \mathrm{Mpc}$ at $\mathrm{z}=1$ in redshift space (although the $10 \%$ accuracy is reached at $k<0.4 \mathrm{~h} / \mathrm{Mpc}$ at $\mathrm{z}=0$ ).

- The matter field produced by the methods, visible in Figure 1, shows that higher LPT orders produce puffier structures, and this makes it evident why the improvement in the matter density field is poor, but not why the clustering of DM halos is better recovered. Clearly, halo reconstruction is acting here as an optimal smoothing scheme, where the average is done exactly over the patch of matter that has undergone gravitational collapse. Besides, Figure 8 shows that the location of large scale structure is better reproduced by higher order LPT.

- Truncation appears to worsen the performances of LPT in all the probes explored in this work. This is no real news: the original paper [16] used scale-free, power-law power spectra with varying slope, and showed, using cross-correlation of density fields, that 
truncation helps to recover the matter power spectrum of an N-body simulation only for relatively flat spectral indices, $n>-1$; however, no advantadge was reported for steeply declining power spectra, $n=-2$. The $\Lambda \mathrm{CDM}$ power spectrum at the non-linearity scale $k \sim 0.5-1 h / \mathrm{Mpc}$ is even steeper than $n=-2$.

- The Augmentation is effective at focusing the structures, limiting the puffiness caused by the plain LPT displacements. When quantifying its performance, it appears that Augmentation improves the reproduction of the halo power spectrum in real space, but does not show important improvements for other probes. In the power spectrum of matter density field, the Augmentation is very effective when applied to 2LPT, dropping below the $10 \%$ accuracy at higher wavelengths than A3LPT. Similarly, when considering the halo power spectrum in real space, the Augmentation brings noticeable improvements when coupled with $2 \mathrm{LPT}$.

The marginal improvement of Augmentation when applied to 3LPT with respect to when it is applied to $2 \mathrm{LPT}$ is mostly due to the smaller smoothing radius adopted (4 Mpc/h for A2LPT and $1.25 \mathrm{Mpc} / \mathrm{h}$ for A3LPT at $z=0$ ), so that clustering at intermediate scales is dominated by the LPT term.

- MUSCLE provides qualitatively the same improvements brought by the augmentation, although it is much more expensive than the Augmented counterpart in terms of computing time.

- COLA generally outperforms LPT methods, with average differences in the position of halos with respect to that of the N-body amounting to less than $10 \%$ of the interparticle distance, and velocities accurate to within a few per cent (and aligned to within 1-2 degrees). Also in the phase difference, COLA provides the best agreement with simulations, although not by a large factor, being even comparable with $3 \mathrm{LPT}$ at $\mathrm{z}=1$. In the power spectrum of the matter density field no strong differences are present among the runs with different mesh size, except for the coarsest one, which drops below the $10 \%$ accuracy at $k \simeq 0.5 \mathrm{~h} / \mathrm{Mpc}$. On the other hand, when considering halos, COLA runs with varying meshes show some difference, and the 1024 mesh performs as good as, or even better than, the finer meshes.

- It is worth mentioning that while good accuracy is found for the monopole of the power spectrum in redshift space, so that few per cent accuracy up to $k=0.5 h / \mathrm{Mpc}$ is achieved by several methods, the reconstruction of the quadrupole is subject to larger errors, even for COLA. This is especially true at $z=0$, where the best LPT-based methods lose $10 \%$ of their power already at $k=0.2 \mathrm{~h} / \mathrm{Mpc}$.

The results presented in this paper set upper limits to the ability of these approximate methods to recover the clustering of halos, and point to the conclusion that LPT has an intrinsic accuracy limit, so that it cannot reconstruct power at scales smaller than $k=0.5 h / \mathrm{Mpc}$, even starting from perfect knowledge of DM halos.

This has implications mostly for the "Lagrangian based" methods, as defined in the Introduction and in [10]. But this does not mean that approximate methods cannot be more accurate than this, if a different strategy is adopted. An example in this sense is given by the Patchy code [32], of the "bias-based" class. Here a sophisticated model for the bias is adopted to populate the large-scale density field, and suitable calibration of parameters allows to recover, to within few per cent accuracy, two- and three-point clustering up to 
$k \sim 0.5 \mathrm{~h} / \mathrm{Mpc}$. It has been shown by [33] that some gain in accuracy is achieved by producing the density field with FastPM [26] in place of A2LPT, but this gain is much more modest than the accuracy gain shown here comparing COLA with A2LPT. There is a trade-off here: the stochastic generation of halos implies that the agreement cannot be at the object-by-object level, and parameter calibration, that needs an N-body simulation to calibrate against, must be repeated if the parent halo catalog is changed. So predictivity is compromised in favour of speed and accuracy. This can be a very useful compromise, for instance if a very large number of realizations of a given observed survey are needed, but may be a limit, for instance if one wants to sample a cosmological parameter space with mock catalogs.

\section{Acknowledgments}

Simulations have been run at CINECA, thanks to the convention with Trieste University, and at the Centre for Astrophysics and Supercomputing at Swinburne University of Technology. We acknowledge support from PRIN MIUR () and from Consorzio per la Fisica di Trieste. Data postprocessing and storage has been done on the CINECA facility PICO, granted us thanks to our expression of interest. P.M. and S.B. have been supported by the "InDark" INFN Grant.

\section{References}

[1] Planck Collaboration, P. A. R. Ade, N. Aghanim, M. I. R. Alves, C. Armitage-Caplan, M. Arnaud et al., Planck 2013 results. I. Overview of products and scientific results, A\&A $\mathbf{5 7 1}$ (nov, 2014) A1, [1303.5062].

[2] K. S. Dawson, J.-P. Kneib, W. J. Percival et al., The SDSS-IV Extended Baryon Oscillation Spectroscopic Survey: Overview and Early Data, AJ 151 (feb, 2016) 44, [1508.04473].

[3] M. Levi, C. Bebek, T. Beers, R. Blum, R. Cahn, D. Eisenstein et al., The DESI Experiment, a whitepaper for Snowmass 2013, ArXiv e-prints (aug, 2013) , [1308.0847].

[4] LSST Science Collaboration, P. A. Abell, J. Allison, S. F. Anderson, J. R. Andrew, J. R. P. Angel et al., LSST Science Book, Version 2.0, ArXiv e-prints (dec, 2009) , [0912.0201].

[5] R. Laureijs, J. Amiaux, S. Arduini, J. . Auguères, J. Brinchmann, R. Cole et al., Euclid Definition Study Report, ArXiv e-prints (oct, 2011) , [1110.3193].

[6] K. Heitmann, M. White, C. Wagner, S. Habib and D. Higdon, The Coyote Universe. I. Precision Determination of the Nonlinear Matter Power Spectrum, ApJ 715 (may, 2010) 104-121, [0812.1052].

[7] D. S. Reed, R. E. Smith, D. Potter, A. Schneider, J. Stadel and B. Moore, Towards an accurate mass function for precision cosmology, MNRAS 431 (may, 2013) 1866-1882, [1206.5302].

[8] A. Schneider, R. Teyssier, D. Potter, J. Stadel, J. Onions, D. S. Reed et al., Matter power spectrum and the challenge of percent accuracy, ArXiv e-prints (mar, 2015), [1503.05920].

[9] D. Potter, J. Stadel and R. Teyssier, PKDGRAV3: Beyond Trillion Particle Cosmological Simulations for the Next Era of Galaxy Surveys, ArXiv e-prints (sep, 2016) , [1609.08621].

[10] P. Monaco, Approximate Methods for the Generation of Dark Matter Halo Catalogs in the Age of Precision Cosmology, Galaxies 4 (Oct., 2016) 53, [1605.07752].

[11] C.-H. Chuang, C. Zhao, F. Prada, E. Munari et al., nIFTy cosmology: Galaxy/halo mock catalogue comparison project on clustering statistics, MNRAS 452 (sep, 2015) 686-700, [1412.7729]. 
[12] C.-H. Chuang, F.-S. Kitaura, F. Prada, C. Zhao and G. Yepes, EZmocks: extending the Zel'dovich approximation to generate mock galaxy catalogues with accurate clustering statistics, MNRAS 446 (jan, 2015) 2621-2628, [1409.1124].

[13] F. Moutarde, J.-M. Alimi, F. R. Bouchet, R. Pellat and A. Ramani, Precollapse scale invariance in gravitational instability, ApJ 382 (dec, 1991) 377-381.

[14] T. Buchert and J. Ehlers, Lagrangian theory of gravitational instability of Friedman-Lemaitre cosmologies - second-order approach: an improved model for non-linear clustering, MNRAS 264 (sep, 1993) 375-387.

[15] P. Catelan, Lagrangian dynamics in non-flat universes and non-linear gravitational evolution, MNRAS 276 (sep, 1995) 115-124, [astro-ph/9406016].

[16] P. Coles, A. L. Melott and S. F. Shandarin, Testing approximations for non-linear gravitational clustering, MNRAS 260 (feb, 1993) 765-776.

[17] A. L. Melott, T. Buchert and A. G. Weib, Testing higher-order Lagrangian perturbation theory against numerical simulations. 2: Hierarchical models, A\&广A 294 (feb, 1995) 345-365, [astro-ph/9404018].

[18] F.-S. Kitaura and S. Heß, Cosmological structure formation with augmented Lagrangian perturbation theory, MNRAS 435 (aug, 2013) L78-L82, [1212.3514].

[19] M. C. Neyrinck, Truthing the stretch: non-perturbative cosmological realizations with multiscale spherical collapse, MNRAS 455 (jan, 2016) L11-L15, [1503.07534].

[20] S. Tassev, M. Zaldarriaga and D. J. Eisenstein, Solving large scale structure in ten easy steps with COLA, JCAP 6 (jun, 2013) 036, [1301.0322].

[21] J. Koda, C. Blake, F. Beutler, E. Kazin and F. Marin, Fast and accurate mock catalogue generation for low-mass galaxies, MNRAS (apr, 2016) , [1507.05329].

[22] A. L. Melott, Comparison of dynamical approximation schemes for nonlinear gravitaional clustering, ApJ 426 (may, 1994), [astro-ph/9402057].

[23] F. Bernardeau, The nonlinear evolution of rare events, ApJ 427 (may, 1994) 51-71, [astro-ph/9311066].

[24] R. W. Hockney and J. W. Eastwood, Computer Simulation Using Particles. 1981.

[25] J. Barnes and P. Hut, A Hierarchical O(NlogN) Force-Calculation Algorithm, Nature 324 (Dec., 1986) 446-449.

[26] Y. Feng, M.-Y. Chu and U. Seljak, FastPM: a new scheme for fast simulations of dark matter and halos, ArXiv e-prints (mar, 2016), [1603.00476].

[27] C. Howlett, M. Manera and W. J. Percival, L-PICOLA: A parallel code for fast dark matter simulation, Astronomy and Computing 12 (sep, 2015) 109-126, [1506.03737].

[28] A. Izard, M. Crocce and P. Fosalba, ICE-COLA: Towards fast and accurate synthetic galaxy catalogues optimizing a quasi N-body method, MNRAS (apr, 2016) , [1509.04685].

[29] V. Springel, S. D. M. White, A. Jenkins, C. S. Frenk, N. Yoshida, L. Gao et al., Simulations of the formation, evolution and clustering of galaxies and quasars, Nature 435 (jun, 2005) 629-636, [astro-ph/0504097].

[30] E. Munari, P. Monaco, E. Sefusatti, E. Castorina, F. G. Mohammad, S. Anselmi et al., Improving the prediction of dark matter halo clustering with higher orders of Lagrangian Perturbation Theory, submitted to MNRAS (2016) .

[31] E. Sefusatti, M. Crocce, R. Scoccimarro and H. M. P. Couchman, Accurate estimators of correlation functions in Fourier space, MNRAS 460 (Aug., 2016) 3624-3636, [1512.07295]. 
[32] F.-S. Kitaura, H. Gil-Marín, C. G. Scóccola, C.-H. Chuang, V. Müller, G. Yepes et al., Constraining the halo bispectrum in real and redshift space from perturbation theory and non-linear stochastic bias, MNRAS 450 (jun, 2015) 1836-1845, [1407.1236].

[33] M. Vakili, F.-S. Kitaura, Y. Feng, G. Yepes, C. Zhao, C.-H. Chuang et al., Accurate halo-galaxy mocks from automatic bias estimation and particle mesh gravity solvers, ArXiv e-prints (Jan., 2017), [1701.03765]. 

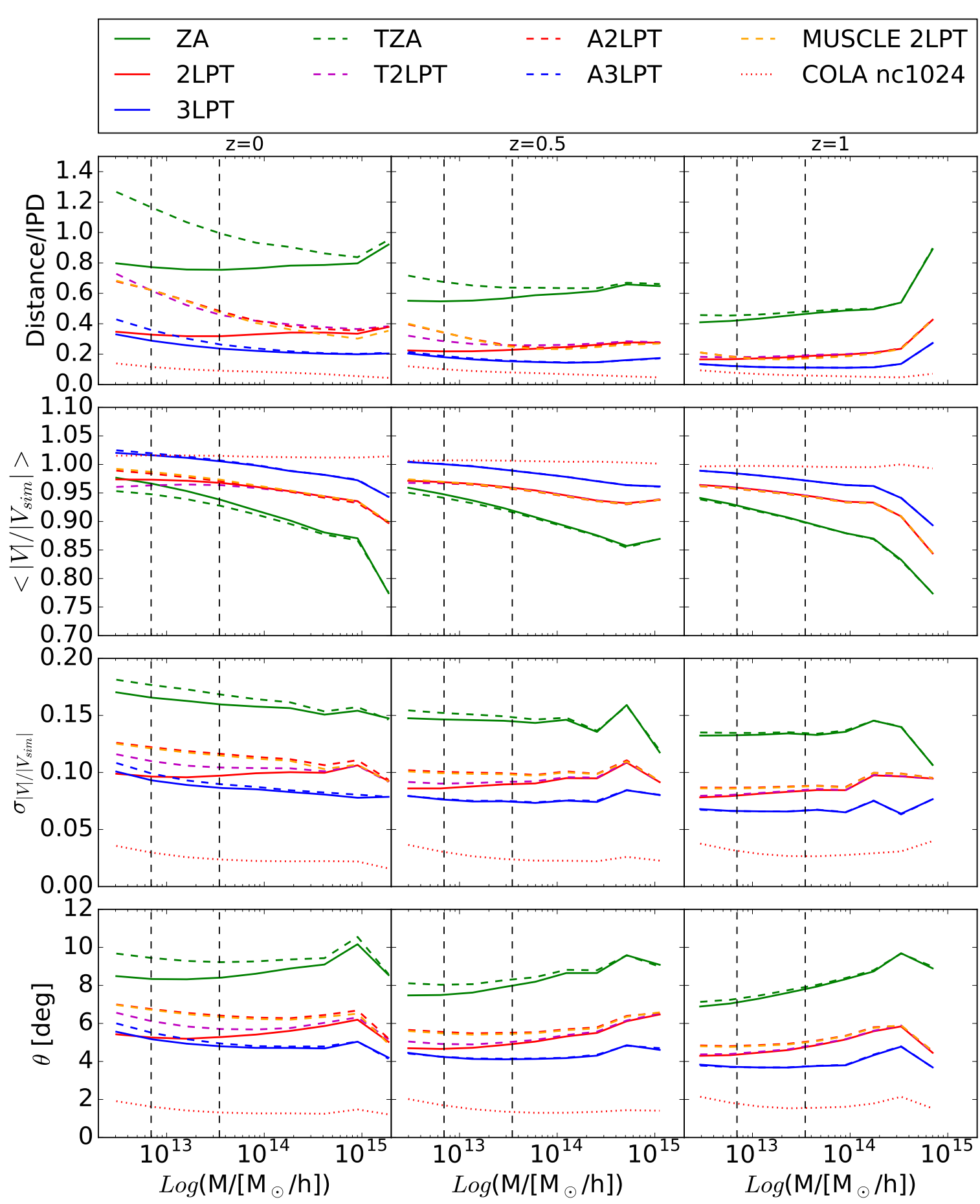

Figure 2. Distance (in units of interparticle distance) and velocity difference at $\mathrm{z}=0$ (panels on the left), 0.5 (panels in the central column), and 1 (panels on the right) of method halos and $N$-body halos. In the top row the quantity $\sqrt{\left\langle(\Delta X)^{2}+(\Delta Y)^{2}+(\Delta Z)^{2}\right\rangle}$ is shown, where $\Delta X, \Delta Y$, and $\Delta Z$ denote the distances, along the three axes, of method halos from the corresponding $N$-body halos. In the second and third row the median values and the dispersion of $|V| /\left|V_{\text {sim }}\right|$ are shown, where $|V|$ denotes the magnitude of the velocity of method halos, and $\left|V_{\text {sim }}\right|$ that of the corresponding $N$-body halos. In the last row the median value of the angle between the velocity vector of method halos and $N$-body halosis shown. The vertical dashed lines identify the mass corresponding to 100 and 500 particles. 


\begin{tabular}{|c|c|c|c|c|c|}
\hline - NBody & $-\cdots \mathrm{TZA}$ & --- & A3LPT & $\ldots \ldots$ & COLA nc2048 \\
\hline$-\mathrm{ZA}$ & -.. T2LPT & $\ldots \ldots .$. & COLA nc512 & & COLA nc3072 \\
\hline$-2 \mathrm{LPT}$ & $\cdots \quad A 2 L P T$ & $\ldots \ldots .$. & COLA nc1024 & -- & MUSCLE 2LPT \\
\hline - $3 \mathrm{LPT}$ & & & & & \\
\hline
\end{tabular}

Matter

Halos
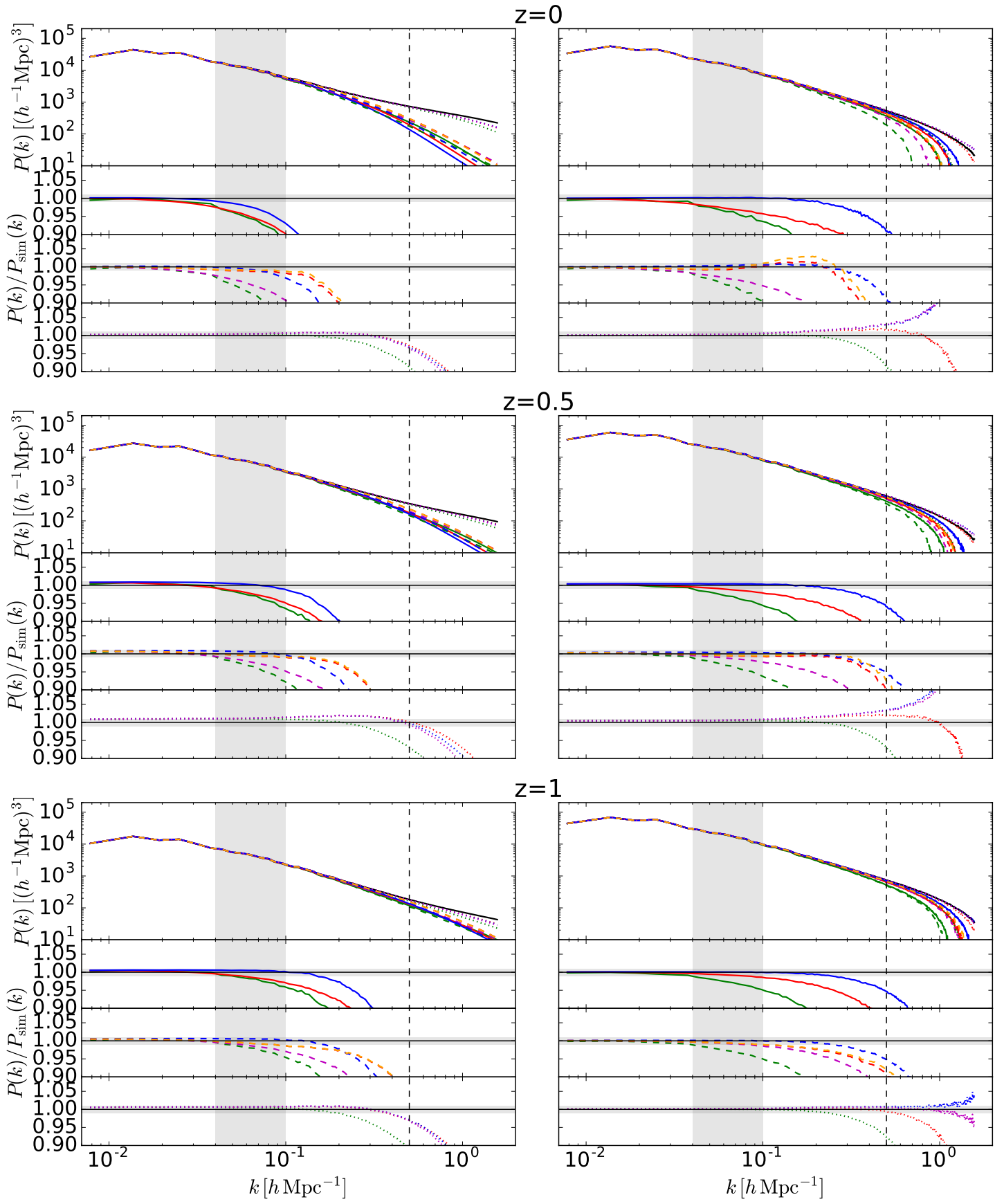

Figure 3. Power spectrum at $z=0,0.5$ and 1 (top, middle and bottom panels, respectively) in real space and ratio with the N-body's one for the matter field (left panels) and for the halo catalogues (right panels). The vertical dashed line locates the $k=0.5 \mathrm{~h} / \mathrm{Mpc}$ where the one-halo term becomes significant. The vertical shaded area locates the region of the BAO peak, while the horizontal one locates the $1 \%$ accuracy region. Part of the results presented in this figure was anticipated in [10]. 


\begin{tabular}{|c|c|c|c|c|}
\hline - NBody & $\ldots$ TZA & $\begin{array}{ll}-\cdots & \text { A3LPT }\end{array}$ & $\ldots \ldots .$. & COLA nc2048 \\
\hline - ZA & $\ldots$ T2LPT & COLA nc512 & & COLA nc3072 \\
\hline$-2 \mathrm{LPT}$ & $\begin{array}{ll}\cdots & \text { A2LPT }\end{array}$ & COLA nc1024 & -- & MUSCLE 2LPT \\
\hline - $3 \mathrm{LPT}$ & & & & \\
\hline
\end{tabular}

$P_{0}$ Halos
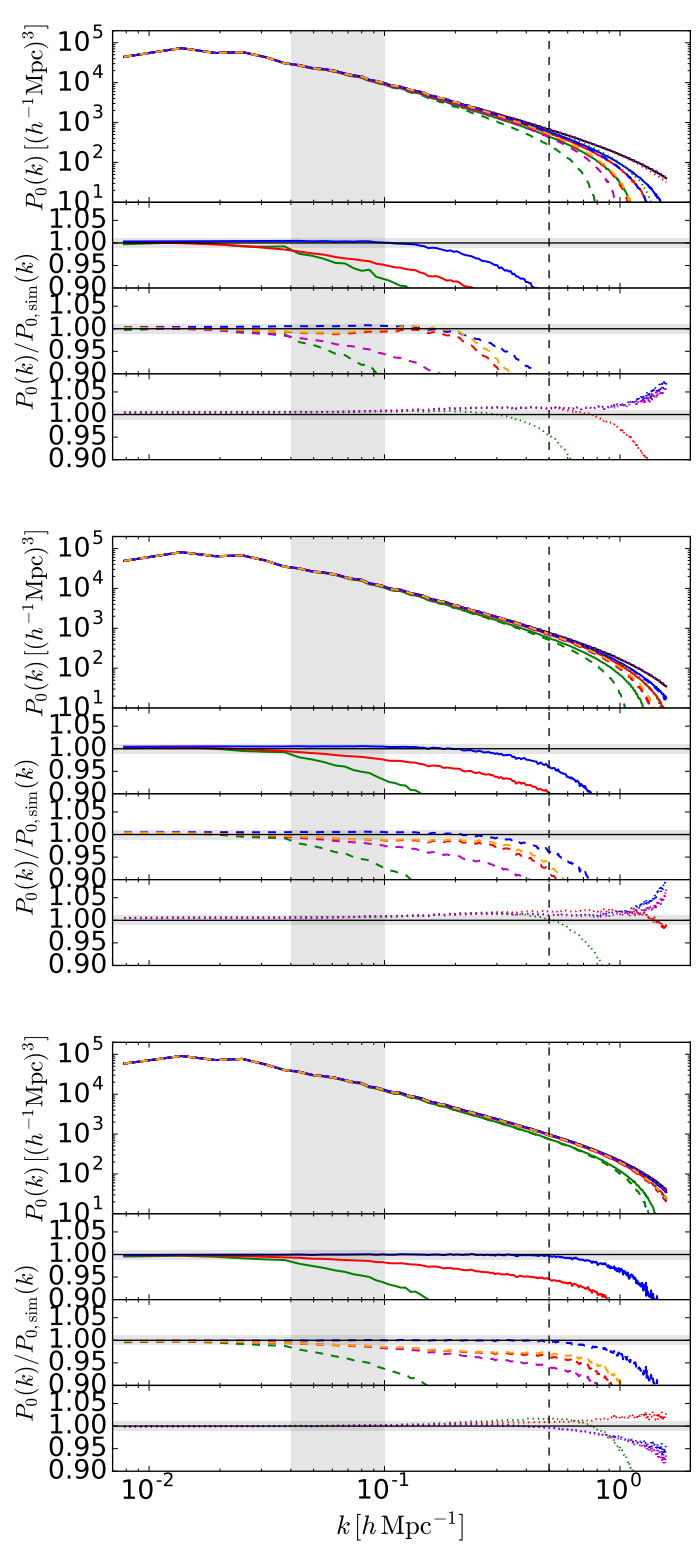

$P_{2}$ Halos

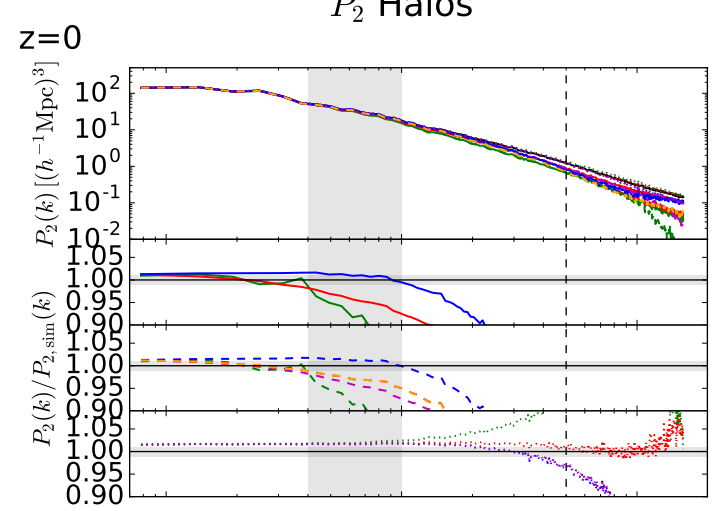

$z=0.5$

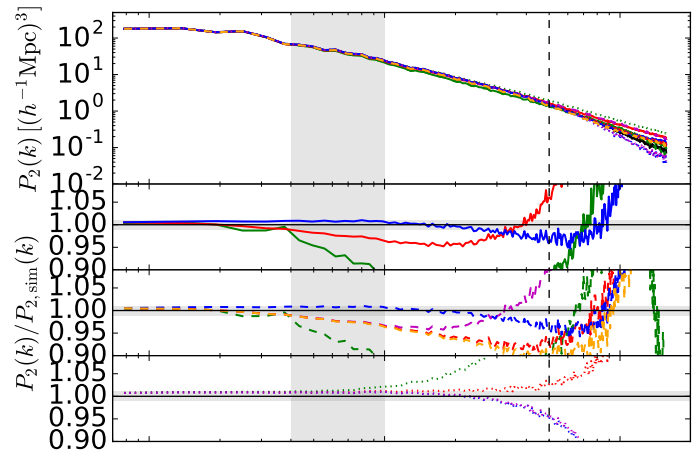

$\mathrm{z}=1$

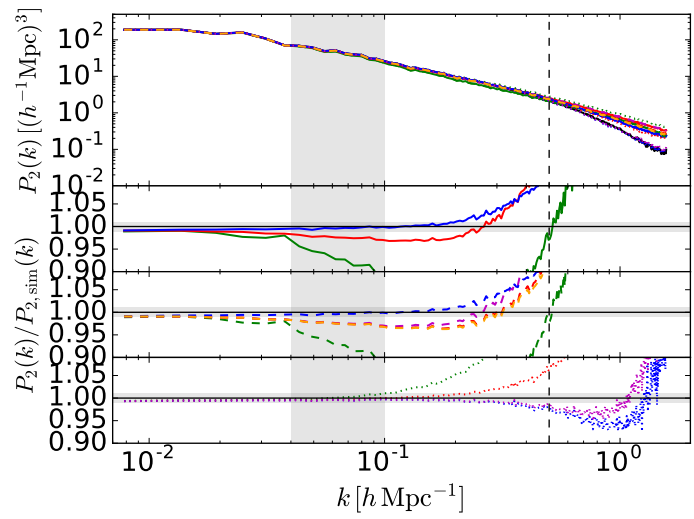

Figure 4. Monopole (left panels) and quadrupole (right panels) of the power spectrum i redshift space at $z=0,0.5$ and 1 (top, middle and bottom panels, respectively), and ratio with the N-body's one for the halo catalogues. The vertical dashed line locates the $k=0.5 h / \mathrm{Mpc}$ where the one-halo term becomes significant. The vertical shaded area locates the region of the BAO peak, while the horizontal one locates the $1 \%$ accuracy region. 


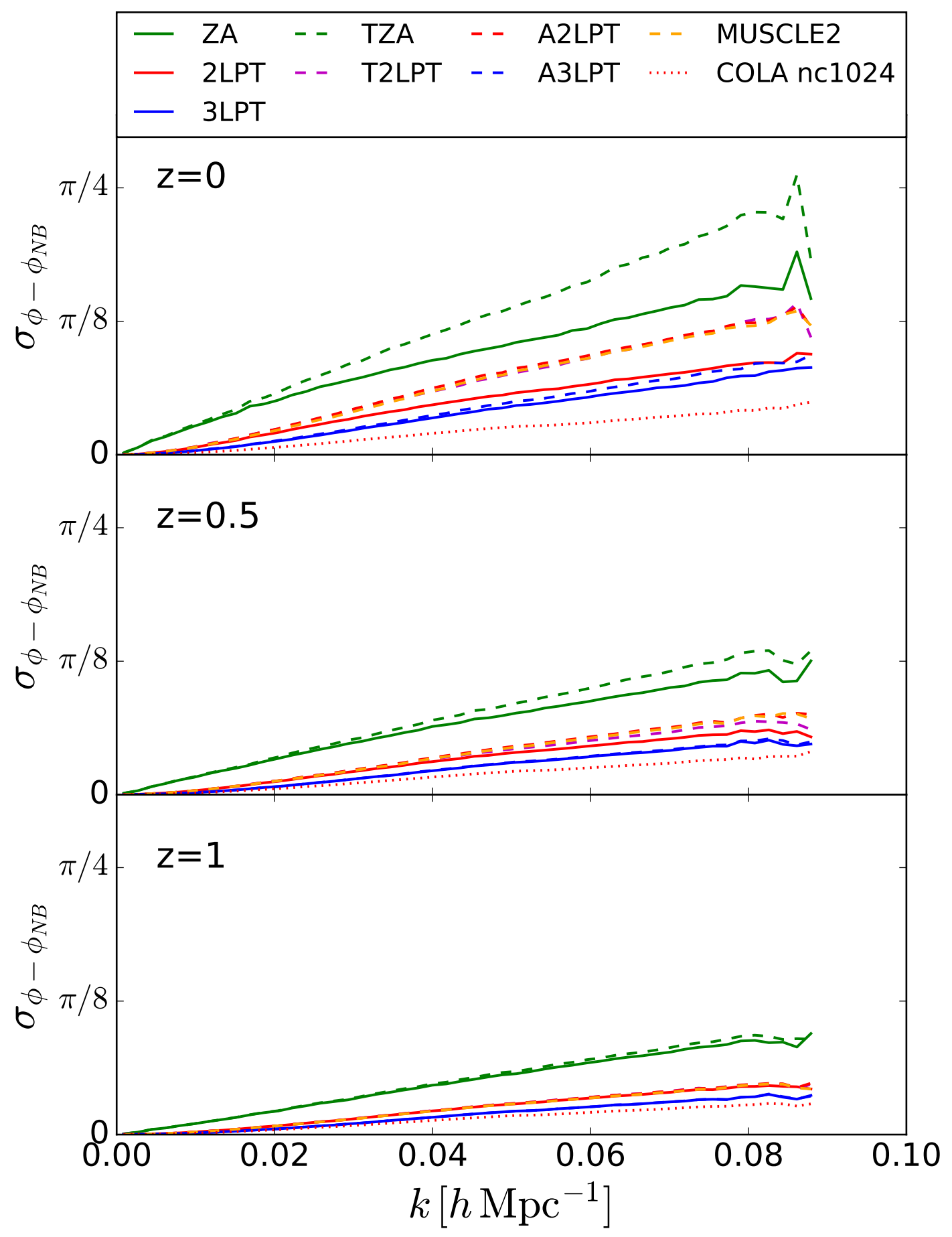

Figure 5. Variance at $\mathrm{z}=0$ (top panel), 0.5 (middle panel) and 1 (bottom panel) of the phase difference between the $N$-body halos catalogue and the method halos catalogues, as indicated in the legend. Phases are computed on a $150^{3}$ cell grid. 

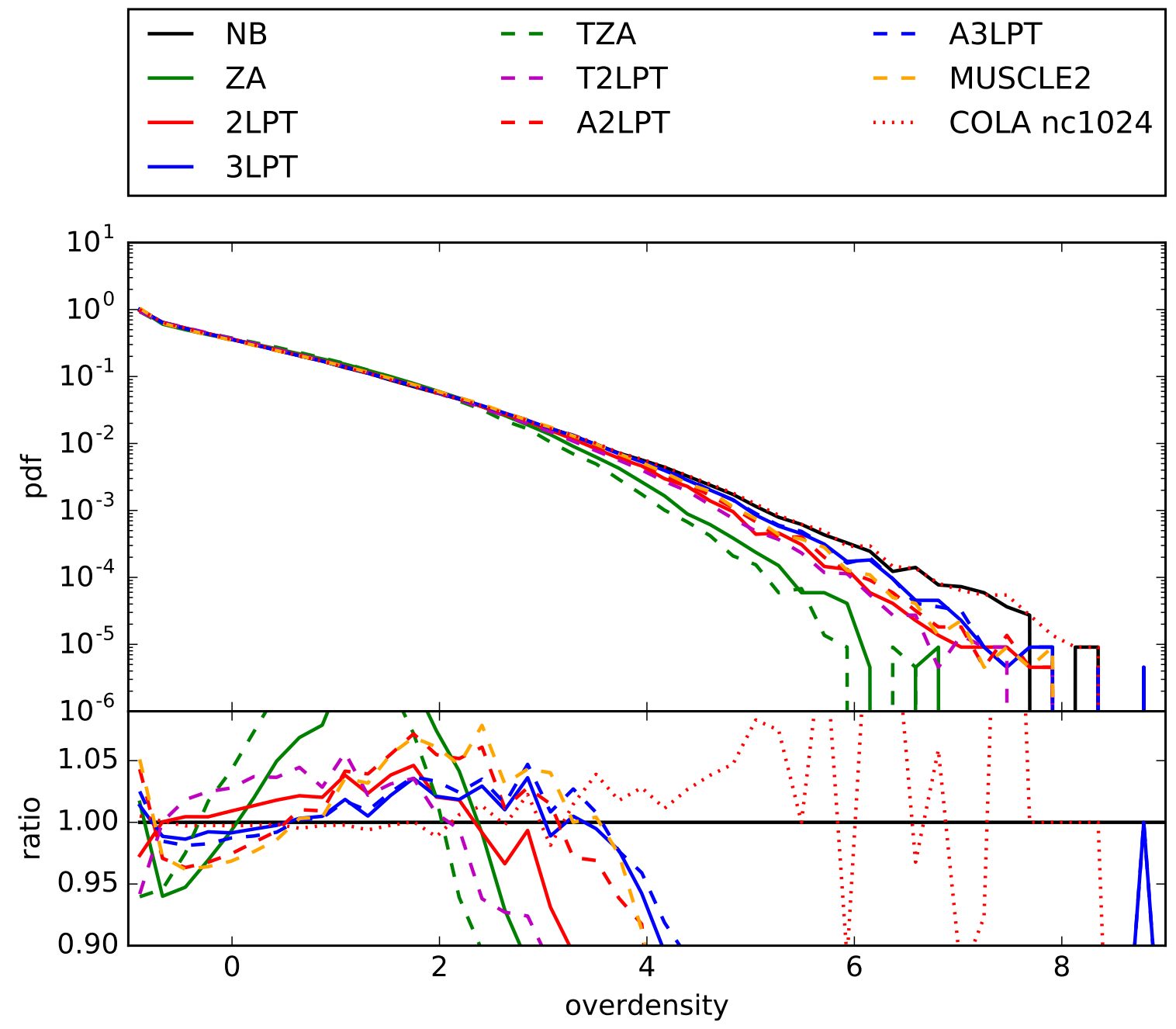

Figure 6. PDF, and ratio with that of the N-body, of the density contrast of the halo distributions, computed by adopting a CIC algorithm on a $100^{3}$ cell grid. 


\begin{tabular}{|llllll|}
\hline NB & $\cdots$ & TZA & $\cdots$ & A3LPT \\
- & ZA & $\cdots$ & T2LPT & $\cdots$ & MUSCLE2 \\
- & 2LPT & $\cdots$ & A2LPT & $\cdots \cdots$ & COLA nc1024 \\
- & 3LPT & $\cdots$ & & & \\
\hline
\end{tabular}
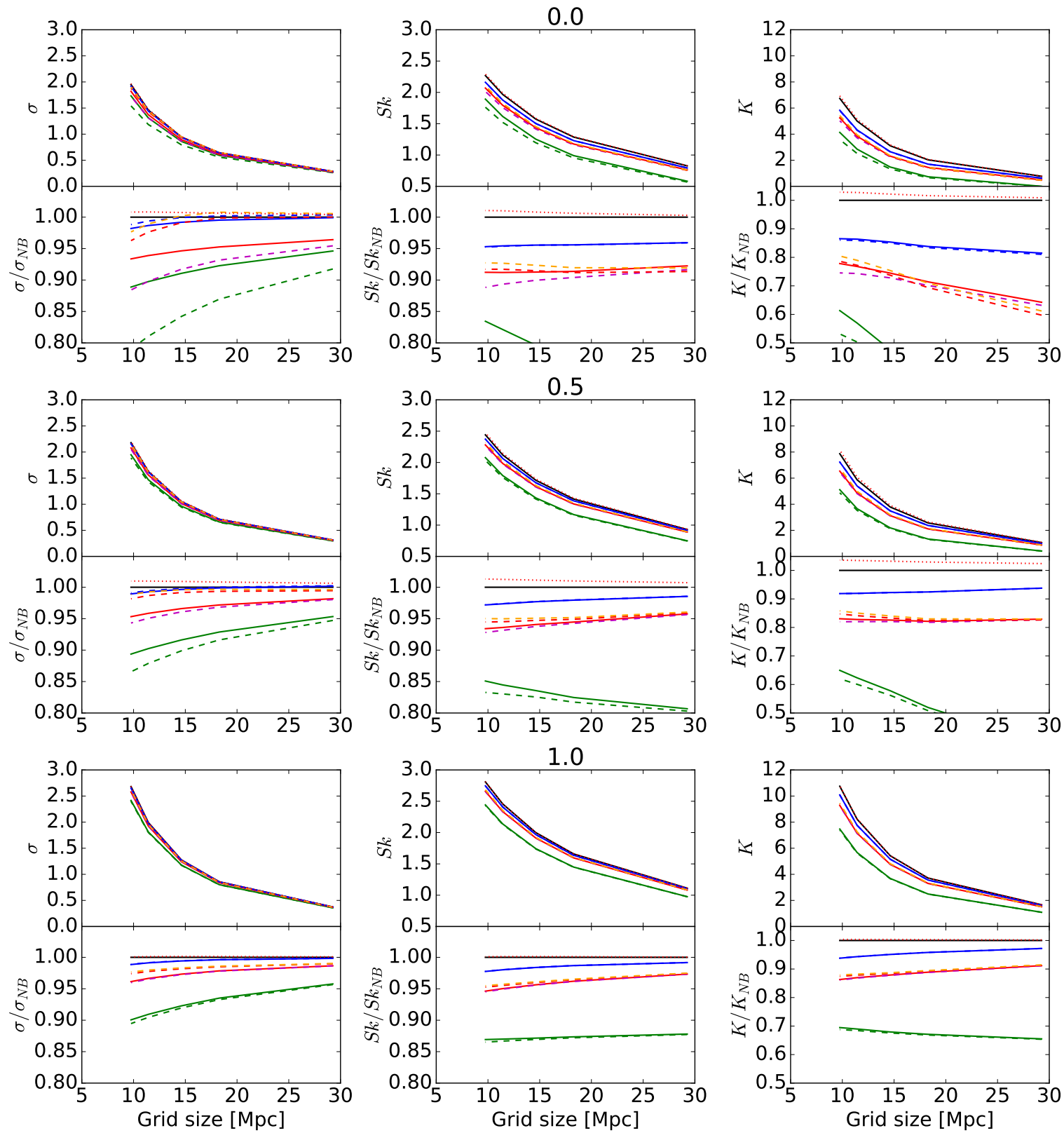

Figure 7. Moments of the PDF (variance in the left column, skewness in the central column, and kurtosis in the right column), and their ratio with that of the N-body,as a function of the grid cell size. The results for $z=0$ are shown in the top row, for $z=0.5$ in the middle row, and $z=1$ in the bottom row. 


\section{Nbody}

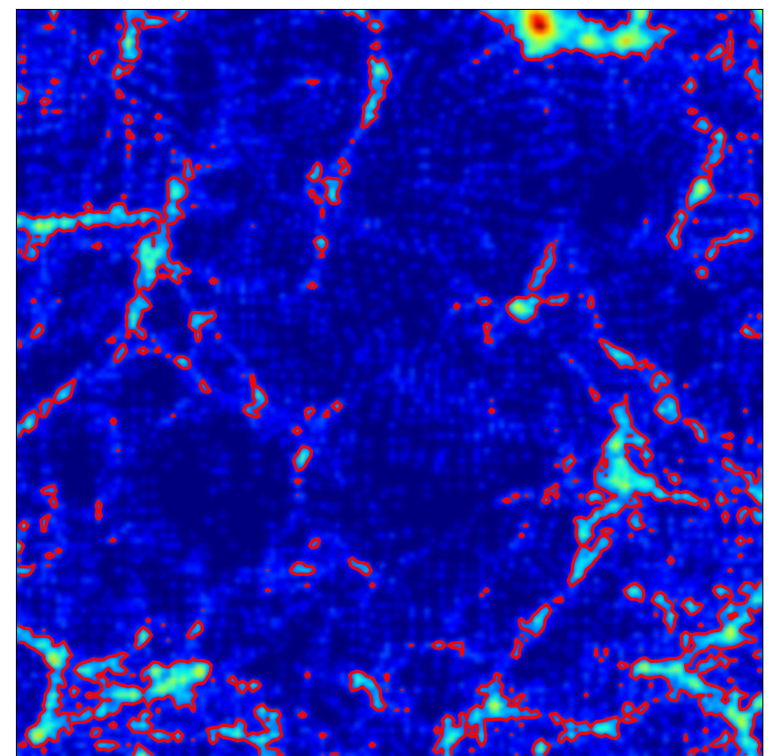

ZA

$3 \mathrm{LPT}$

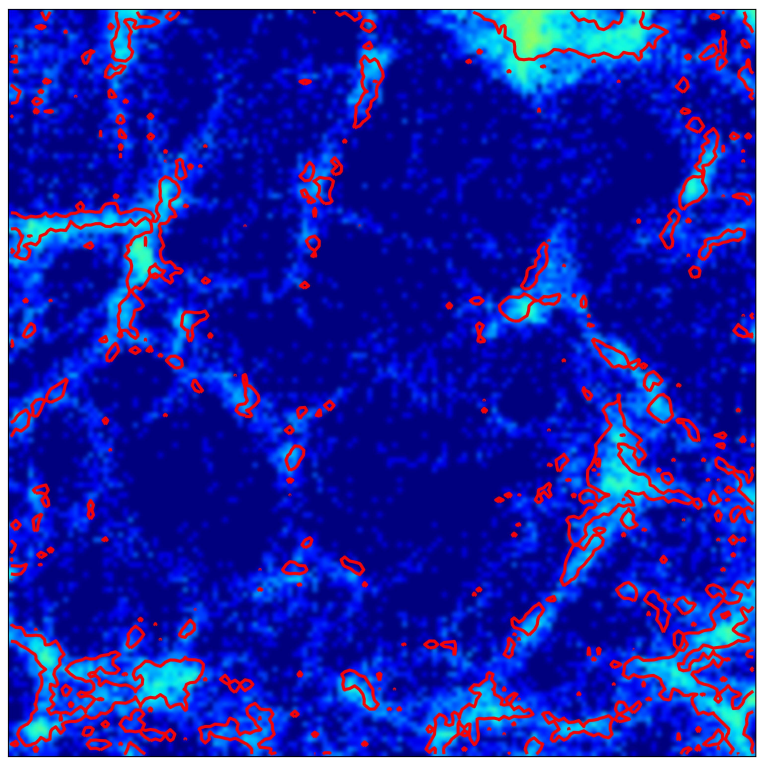

COLA
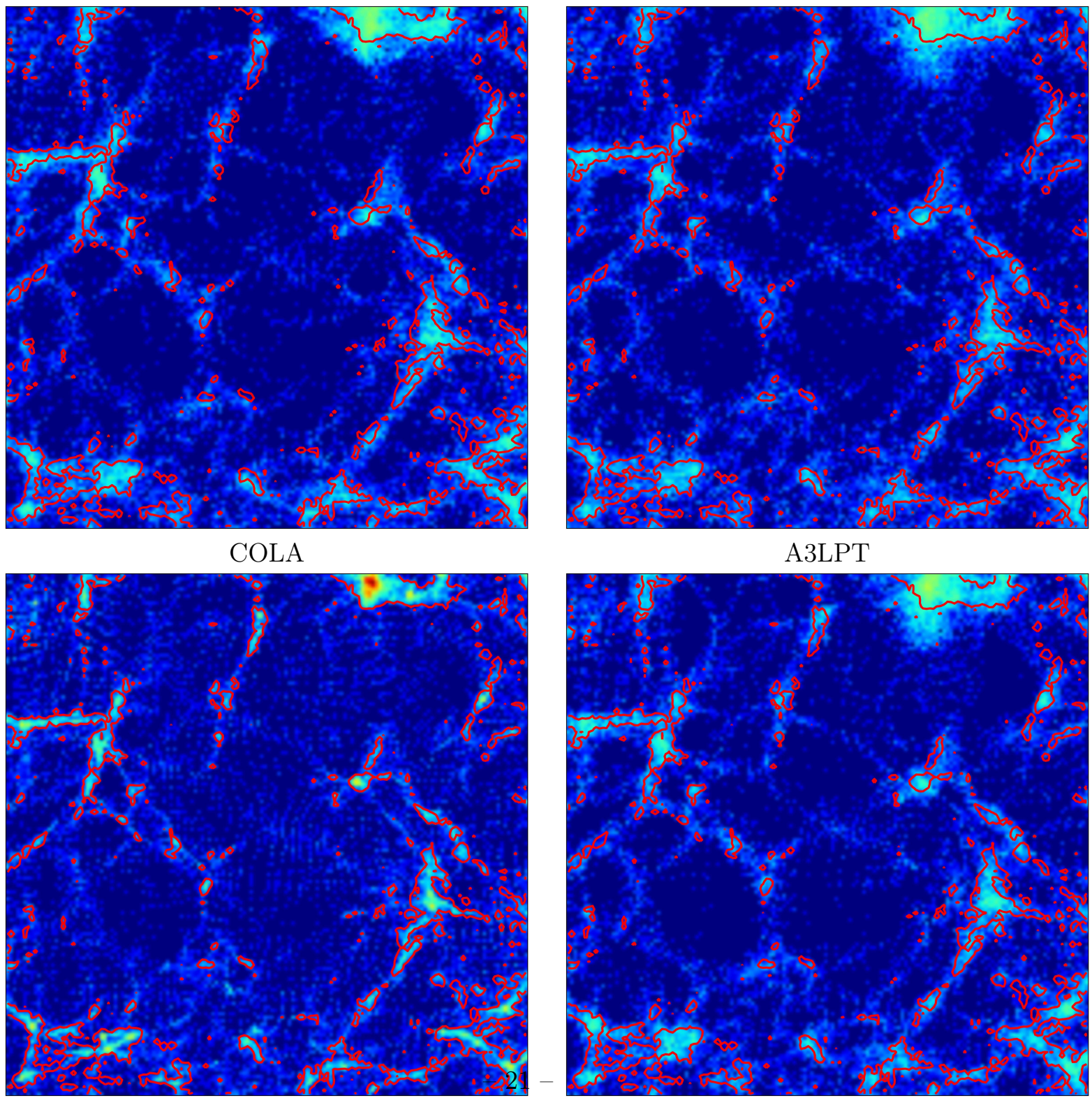

A3LPT

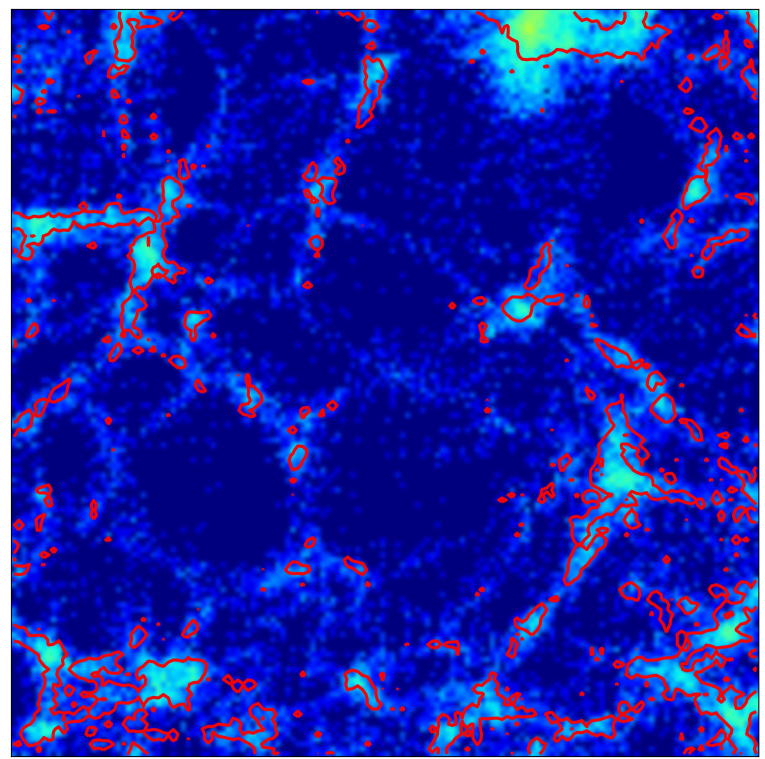

Figure 8. $100 \times 100 \times 10 \mathrm{Mpch}^{-1}$ slice with the density field of the N-body, ZA, 3LPT, COLA, and A3LPT realizations. Color scale is logarithmic. Red lines are the density levels of the N-body realization corresponding to a density threshold equal to 1.5 times the mean density, smoothed with 